Short reports

\title{
Regression of cardiac involvement by malignant melanoma following lomustine chemotherapy
}

\author{
MR Carpenter, M Vassallo, IM Peat, AJI Scriven
}

\begin{abstract}
Summary
Cardiac involvement by malignant melanoma is usually a premorbid event. We present a case of presumed cardiac involvement by malignant melanoma with regression following chemotherapy as demonstrated by transoesophageal echocardiography.
\end{abstract}

Keywords: malignant melanoma, transoesophageal echocardiography, metastatic heart disease

Cardiac deposits are found in $60 \%$ of patients with metastatic malignant melanoma at autopsy. ${ }^{1}$ In life, the finding of cardiac involvement usually occurs in the final stages of the disease, although it is rarely the cause of death. ${ }^{2-4}$ We present a case of intracardiac malignant melanoma diagnosed in life by transoesophageal echocardiography (TOE) which showed initial regression after lomustine chemotherapy, as assessed by TOE.

\section{Case report}

A 53-year-old woman underwent wide local excision and skin grafting of a histologically proven $12-\mathrm{mm}$ thick malignant melanoma on the left elbow. At this time there was no evidence of local or systemic spread.

She presented to medical out-patients 17 months later with a three-month history of chest pain and dyspnoea on exertion. Examination revealed no clinical evidence of metastatic disease but a resting electrocardiogram (ECG) showed $\mathrm{T}$-wave changes and reduced $\mathrm{R}$-wave amplitude in the anterior chest leads consistent with a diagnosis of myocardial ischaemia. She underwent treadmill exercise testing and completed eight minutes of the modified Bruce protocol, with no ECG changes or chest pain. Subsequent echocardiography (Hewlett Packard Sonos 1500, 2.5 $\mathrm{MHz}$ transducer) showed gross thickening of the intraventricular septum $(3.2 \mathrm{~cm})$, and the presence of a mobile mass within the right ventricle. To further define this mass TOE was performed. This demonstrated the distal septum thickening seen before (figure 1), and two masses in the right ventricle, one adjacent to a papillary muscle and a further frond-like mass was seen in the body of the ventricle, extending into the outflow tract and reducing the infundibular diameter by $50 \%$. No further masses were seen in the right atrium or pulmonary arteries.
Ultrasound scanning and chest radiography showed no evidence of renal, lung or intraabdominal pathology. Bone scanning showed widespread increased uptake in the right humerus, several ribs, femoral shaft, and the skull vault. Plain radiography of the affected bones confirmed the presence of lytic metastases. Biopsy of the right ventricular mass was considered but was rejected because of the risk of complications. The presumed diagnosis of cardiac involvement by malignant melanoma was made on the basis of the widespread metastatic disease and the particular echocardiographic findings (intramyocardial deposits) which are usually only found in lymphomatous or melanomatous cardiac deposits. ${ }^{5}$ The patient received a single fraction of radiotherapy to the shoulder and four courses of oral lomustine chemotherapy at six-to-eight weekly intervals. After the first course she became pain free.

Repeat TOE (figure 2) following the final course of chemotherapy showed marked reduction of septal thickness and no evidence of either of the two right ventriculer masses. The patient remained in clinical remission for 11 months. Back pain then recurred and she developed new skin nodules. There were no cardiac symptoms or signs but repeat transthoracic echo showed the presence of areas of new myocardial thickening consistent with myocardial recurrence. The patient deteriorated with increasing dyspnoea and died four months later. Post mortem examination revealed widespread evidence of metastatic malignancy with no evidence of a second primary. Examination of the heart showed numerous metastases in the epicardium and myocardium, one

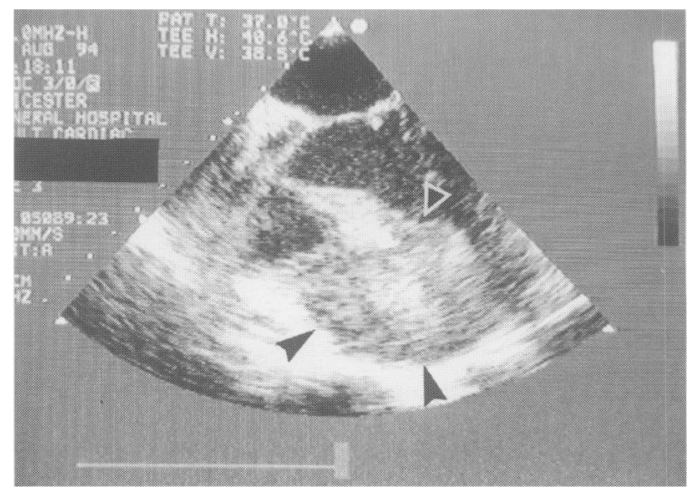

Figure 1 TOE image of the intraventricular septum before chemotherapy showing considerable thickening of the distal septum (arrows) 


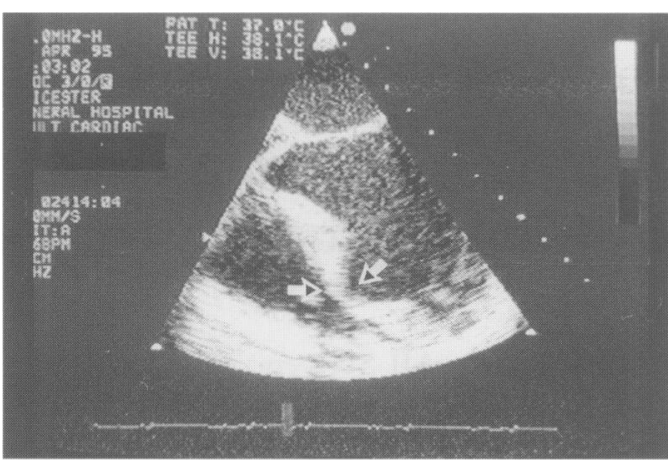

Figure 2 Repeat TOE image following chemotherapy showing marked reduction of intraventricular septal thickness consistent with regression of the melanomatous deposits (arrows)

of which corresponded to the site of the deposit seen on TOE. In addition there were several tumour nodules seen in the left ventricular outflow tract and proximal pulmonary artery.

\section{Discussion}

Symptomatic cardiac involvement of the heart by malignant melanoma is usually a premorbid event, although cerebral haemorrhage or widespread metastatic disease rather than cardiac involvement are usually the cause of death. ${ }^{1}$ The most frequent sites of cardiac involvement in malignant disease are the pericardium and epicardium. Myocardial involvement is rare and is usually associated with cutaneous malignancies and lymphomata.

Symptoms and signs of cardiac involvement in malignant melanoma are generally nonspecific with many common cardiac signs being caused by metastatic disease. Dyspnoea can be caused by pulmonary metastases, chest pain by bony metastases, and raised jugular venous pressure by superior vena caval obstruction. In an autopsy study of 70 patients with malignant melanoma there was no differ-

1 Glancy DL, Roberts WC. The heart in malignant melanoma a study of 70 autopsy cases. Am 7 Cardiol 1968; 21: 555-71.

2 Schneider B, Zienkiewicz T, Langenstein B, et al. Metastatic malignant melanoma initially seen as congestive heart failure: diagnosis by transoesophageal echocardiography. Am Heart f 1994; 128: 414-6.

3 Lustig V, Vlasveld LT, Bakker RH, et al. Intracardiac metastases, report of three cases. Neth $\mathcal{F}$ Med 1991; 38: 2932 .

\section{Summary points}

- metastatic disease should be considered in any patient with a previous diagnosis of malignant melanoma who presents with atypical symptoms or signs

- symptoms and signs of cardiac involvement often mimic those of other features of malignant disease, eg, dyspnoea, chest pain

- ECG changes in cardiac involvement are nonspecific and easily confused with the changes of ischaemic heart disease

- the diagnosis of cardiac involvement by malignant melanoma can be made by transthoracic echo, but TOE can add further information

- chemotherapy can induce regression of cardiac involvement which may persist in the medium term

ence in the incidence of the above symptoms or the common ECG abnormalities of cardiac involvement (sinus tachycardia, atrial arrhythmias, low voltage $Q R S$ complexes or repolarisation abnormalities) in the patients with or without cardiac involvement. ${ }^{1}$ However, in the presence of atypical features in any patient with malignant melanoma, cardiac involvement should be considered.

Echocardiography is a noninvasive and welltolerated method of visualising intracardiac anatomy, with a high pick-up rate for the diagnosis of intracardiac tumours. ${ }^{6}$ The better image quality of TOE has been shown to improve the ability to diagnose the nature of intracardiac involvement and may obviate the need for biopsy.

In conclusion, previous reports of cardiac involvement by malignant melanoma have featured autopsy series or patients in the premorbid stages of their disease. Our patient showed regression of the tumour mass following chemotherapy, as demonstrated by TOE, and remained in remission for 11 months after chemotherapy before relapsing clinically and on echocardiography.

4 Kutalek SP, Panidis IP, Kotler MN, et al. Metastatic tumours of the heart detected by 2D echocardiography. Am Heart f 1985; 109: 343-9.

5 MacGee W. Metastatic and invasive tumours of the heart in a geriatric population: a necropsy study. Virch Arch A Pathol Anat Histopathol 1991; 419: 183-9.

6 Engberging R, Daniel WG, Erbel R, et al. Diagnosis of heart tumours by transoesophageal echocardiography: a multitumours by transoesophageal echocardiography: a multi- 